\title{
Independent domains of disoriented chiral dondensate
}

\author{
Q. H. Zhang \\ Institüt für Thoretisch Physik, Universität Regensburg, D-93040 Regensburg, Germany \\ X. Q. Li \\ China Center of Advanced Science and Technology(World Laboratory), P.O. Box 8730, Beijing \\ 100080, P.R. China \\ Physics Department, Nankai University, Tianjing 300071, China
}

\begin{abstract}
The probability distribution of a neutral pion fraction from independent domains of disoriented chiral condensate is characterized. The signal for the condensate is still clear for a large number of independent domains if one of them is predominant.
\end{abstract}


A disoriented dhiral condensate (DCC) may be formed in large hot regions of hadronic matter where the approximate chiral symmetry of QCD has been briefly restored. The decay of this region induces a non-equilibrium relaxation of the chiral fields which is predicted to create coherent sources of soft pion modes [1]. The charge of the pions emitted from the DCC has a characteristic probability distribution [2, 3]

$$
P(f)=\frac{1}{2 \sqrt{f}}
$$

where

$$
f=\frac{n_{0}}{n_{\text {total }}} .
$$

Here $n_{0}$ is the number of observed neutral pions while $n_{\text {total }}$ is the total number of observed pions. This is markedly different from the standard statistical distribution which, for large $n_{\text {total }}$, we expect to be

$$
P(f)=\delta\left(f-\frac{1}{3}\right)
$$

Using the discrete wavelet transformation method, Huang et al. [4] calculated the probability distribution of the neutral pion fraction $f$ for different physical scales and found that due to the DCC cluster size, $P(f)$ exhibits a delay in approaching Eq.(3) required by the central limit theorem (delayed central limit). This can be used as a signature of DCC in high energy heavy-ion collisions.

Recently Amado and $\mathrm{Lu}$ [5] claimed that for more than three independent domains of DCC the signal for condensate (Eq.(1)) will be reduced. But their conclusion is based on the assumption that the $N$ independent regions have equal weight which means that each region emits the same total number of pions. In the case that the number of the regions goes to infinity, the probability distribution of the neutral pion fraction becomes $P(f)=\delta(f-1 / 3)$. In this Brief Report, we will prove that if one of these regions is predominant, that is, if most pions are emitted from one of the uncorrelated domains, we can still have a clear signal for the condensate even though the number of the independent regions is very large. It is 
likely that, particularly in a heavy ion collisions, more than one domain or region of chiral condensate will be formed in the large interaction volume [4]. Similar to ref. [5], we assume that these domains are uncorrelated, that is, pions are emitted independently from each domain. In that case the observed neutral pion fraction $f$ will be an average over these independent regions. It is also likely that each region has a different weight $\alpha_{i}=\frac{n_{i, \text { total }}}{\sum_{i} n_{i, \text { total }}}$. Here $n_{i}$ is the total number of pions emitted from region $i$. In each single region we take the probability of $f$ to be given by $P_{1}(f)$ (eq.(1)). Then the probability of finding a neutral fraction $f$ averaged over the $\mathrm{N}$ regions is given by

$$
P_{N}\left(f, \alpha_{i}\right)=\int d f_{1} \ldots d f_{N} \delta\left(f-\sum_{i=1}^{N} \alpha_{i} f_{i}\right) P_{1}\left(f_{1}\right) P_{1}\left(f_{2}\right) \ldots . . P_{1}\left(f_{N}\right) .
$$

This can be transformed into a recursion relation,

$$
P_{N}\left(f, \alpha_{i}\right)=\frac{1}{1-\alpha_{1}} \int P_{N-1}\left(\frac{f-\alpha_{1} f_{1}}{1-\alpha_{1}}, \frac{\alpha_{i}}{1-\alpha_{1}}\right) P_{1}\left(f_{1}\right) d f_{1} .
$$

This relation is particularly helpful in computing $P_{N}$ stepwise in N. For two domains the probability can be found analytically and we get

$$
P_{2}\left(f, \alpha_{i}\right)=\frac{\pi}{4 \sqrt{\alpha_{1} \alpha_{2}}}
$$

for $f<\min \left\{\alpha_{1}, \alpha_{2}\right\}$,

$$
P_{2}\left(f, \alpha_{i}\right)=\frac{1}{4 \sqrt{\alpha_{1} \alpha_{2}}}\left[\pi-2 \arccos \left(\sqrt{\frac{\min \left\{\alpha_{1}, \alpha_{2}\right\}}{f}}\right)\right]
$$

for $\min \left\{\alpha_{1}, \alpha_{2}\right\} \leq f \leq \max \left\{\alpha_{1}, \alpha_{2}\right\}$ and

$$
P_{2}\left(f, \alpha_{i}\right)=\frac{1}{4 \sqrt{\alpha_{1} \alpha_{2}}}\left[\pi-2 \arccos \left(\sqrt{\frac{\alpha_{1}}{f}}\right)-2 \arccos \left(\sqrt{\frac{\alpha_{2}}{f}}\right)\right]
$$

for $f>\max \left\{\alpha_{1}, \alpha_{2}\right\}$. For the case $\alpha_{1}=\alpha_{2}=1 / 2$ the result was previously calculated in [4.6]. In the following, we will show that for $\alpha_{1}=\alpha_{2}=\cdots=\alpha_{N}=1 / N$, as $N$ tends to infinity, the probability distribution reaches exactly eq.(3). It is easily checked that eq.(4) can be written down as 


$$
\begin{aligned}
G(f) & =\lim _{N \rightarrow \infty} P_{N}(f) \\
& =\frac{1}{2 \pi} \lim _{N \rightarrow \infty} \int d f_{1} d f_{2} \cdots d f_{N} d y e^{i f y-i \frac{f_{1}+\cdots+f_{N}}{N} y} P_{1}\left(f_{1}\right) \cdots P_{1}\left(f_{N}\right) \\
& =\frac{1}{2 \pi} \lim _{N \rightarrow \infty} \int d f_{1} d f_{2} \cdots d f_{N} d y e^{i f y} \sum_{n=0}^{\infty}(-i)^{n} \frac{\left(f_{1}+\cdots+f_{N}\right)^{n}}{N^{n} n !} P_{1}\left(f_{1}\right) \cdots P_{1}\left(f_{N}\right),
\end{aligned}
$$

Using the relationship that

$$
\lim _{N \rightarrow \infty} \int d f_{1} d f_{2} \cdots d f_{N} \frac{\left(f_{1}+\cdots+f_{N}\right)^{n}}{N^{n} n !} P_{1}\left(f_{1}\right) \cdots P_{1}\left(f_{N}\right)=\frac{1}{3^{n} n !}
$$

we have

$$
G(f)=\delta(f-1 / 3)
$$

Now we consider the non equal-weight case and for simplicity we assume that $\alpha_{1}=0.8$, that is, eighty percent of the pions are emitted from one of those independent domains, the other pions are emitted by other regions with weight $\alpha_{2}=\cdots=\alpha_{N}=0.2 /(N-1)$. The numerical results are shown in Fig.1 . For $N \rightarrow \infty$, we have

$$
G(f)=0
$$

for $f \leq 1 / 15$

$$
G(f)=\frac{5}{4} \frac{1}{\sqrt{5 f-1 / 3}}
$$

for $1 / 15 \leq f \leq 13 / 15$ and

$$
G(f)=0
$$

for $13 / 15 \leq f$. This shows clearly the difference between our results and previous results [5]. Even with many independent regions of DCC, we can still have a clear signal of DCC if one of these regions is predominant.

Conclusion: In this Brief report, we calculated the probability distribution of $P(f)$ for more than three independent domains with different weights. It was shown that if one of this DCC regions is predominant, we can still see the signal of the condensate even if the number of domains is very large. 
One of the authors (Q.H.Z.) thank Dr. Stefan Ochs for reading the manuscript. This work was supported in part by the Alexander von Humboldt foundation in Germany and the National Natural Science Foundation of China. 


\section{REFERENCES}

[1] A. A. Anselm, Phys. Lett. B217, 169 (1989),

A. A. Anselm and M. G. Ryskin, Phys. Lett. B266, 482 (1991),

K.Rajagopal and F. Wilczek, Nucl. Phys. B 399, 395(1992); Nucl. Phys. B404,577(1993).

K. Rajagopal in Quark-Gluon Plasma 2ed. R. Hwa, World Scientific (1995)

Z. Huang and X.-N. Wang, Phys. Rev. D49, R4339 (1994),

M. Asakawa, Z. Huang, and X.-N. Wang, Phys. Rev. Lett. 74, 3126 (1995),

A. A. Anselm and M. Bander, Pis'ma Zh. Eskp. Teor. Fiz.59, 479 (1994)[JETP Lett. $59,503(1994)]$;

I. I. Kogan, Phys. Rev. D48, R3971 (1993); Pis'ma Zh. Eksp. Teor. Fiz. 59, 289 (1994)[JETP Lett. 59, 307 (1994)];

S. Gavin, A.Gocksch, and R. D. Pisarski, Phys. Rev. Lett.72, 2143 (1994),

S. Gavin and B. Muller, Phys. Lett. 329B, 486 (1994),

D. Boyanovsky, H. J. de Vega, and R, Holman, Phys. Rev. D 51, 734 (1995),

F. Cooper, Y. Kluger, E. Mottola, and J. P. Paz, Phys. Rev. D 51, 2377 (1995),

A. Bialas, W. Cryz, and M. Gmyrek, Phys. Rev. D51, 3239 (1995),

A. Barducci, L. Caiani, R. Cassalbuoni, M. Mondugnio, G. Pettini, and R. Gatto, Phys. Lett. B369, 23 (1996).

[2] J. P. Blaizot and A. Krzywicki, Phys. Rev. D46,246 (1992);

I.V. Andreev, JETP Lett. 33, 367 (1981).

[3] J. D. Bjorken, Int. J. Mod. Phys. A7 4189 (1992); Acta Phys. Pol. B23, 561 (1992).

[4] Z. Huang, I. Sarcevic, R. Thews, and X.N. Wang, Phys. Rev. D 54, 750 (1996).

[5] R. D. Amado and Y. Lu, Phys. Rev. D 54, 7075 (1996).

[6] R. D. Amado and I. I. Kogan, Phys. Rev. D 51, 190 (1995). 


\section{FIGURE CAPTIONS}

Fig.1 The probability density $P(f)$ vs. neutral pion fraction $f$. 


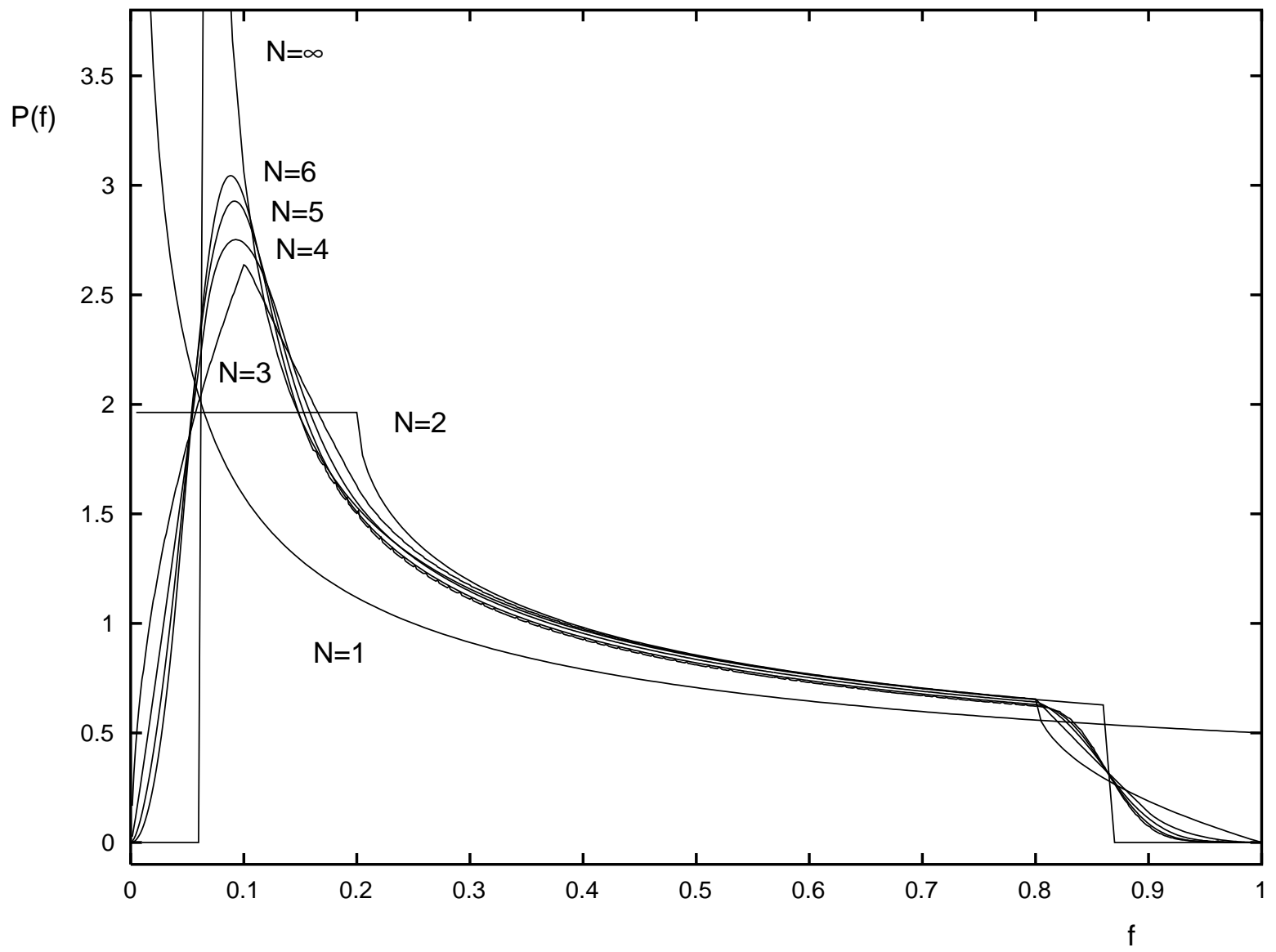

Fig.1

By Q.H. Zhang et. al. 